\title{
Comparison of Tomographic Systems for $X$-Ray and Thermal Neutrons
}

\author{
Maria Ines S. Souza, Gevaldo L. de Almeida, Rosanne C.A.A. Furieri, \\ Instituto de Engenharia Nuclear - CNEN \\ C.P. 68550, Ilha do Fundão \\ 21945-970 Rio de Janeiro, RJ, Brazil
}

\author{
Ricardo Tadeu Lopes, Edgar Oliveira de Jesus, \\ Universidade Federal do Rio de Janeiro, COPPE \\ Centro de Tecnologia, Bloco 6, 21945-970, Rio de Janeiro, RJ, Brazil
}

and Ademarlaudo França Barbosa

Centro Brasileiro de Pesquisas Físicas- CNPq

Rua Xavier Sigaud 150, 22290-180, Rio de Janeiro, RJ, Brazil

Received on 30 October, 2002

\begin{abstract}
In this work, tomographic images of the same object have been taken with $25 \mathrm{keV} \mathrm{X}$-rays and thermal neutrons $(\mathrm{E}=0.025 \mathrm{eV})$ aiming to demonstrate that thermal neutron tomography in some cases is a complementary technique to the X-ray tomography, such as in the examination of hydrogen-bearing compounds wrapped in a metallic matrix for instance. The capability of the neutron to pass through metallic materials such as lead, stainless steel and aluminium, allows to inspect encapsulated plastic explosives and visualize their inner structure like density variations, voids and alien materials, which are important features for the quality control of the final product. To obtain the images, a $3^{\text {rd }}$ generation tomographic system with a Position Sensitive Detector has been developed. For X-rays this proportional detector was provided with an $8 \mathrm{~cm}$ long carbon window, and filled with $\mathrm{Ar}-\mathrm{CH}_{4}$ under a pressure of $2 \mathrm{~atm}$. The X-ray beam was supplied by an ampoule with a tungsten anode manufactured by IPRJ/UERJ. For neutron detection the carbon window has been replaced by aluminium, and the filling-gas by ${ }^{3} \mathrm{He}$ enriched helium, acting simultaneously as neutron converter and ionization gas. The Argonauta reactor at the Instituto de Engenharia Nuclear / CNEN was used as neutron source and furnishes a thermal neutron flux of $4.5 \times 10^{5} \mathrm{n} \cdot \mathrm{cm}^{-2} \cdot \mathrm{s}^{-1}$ at its main channel outlet, where the tomographic system was installed.
\end{abstract}

\section{Introduction}

The radiographic method via transmission is based on the attenuation of the radiation through the matter. On this basis, the inner physical structure of an object can be examined by measuring the transmitted radiation intensity with a proper detector. The attenuation coefficient of the materials depends upon the nature and energy of the incident radiation, and thus, the more suitable radiation and energy will depend upon the involved materials. Important parameters which should be taken into account are the size and composition of the object under inspection, the kind and level of the information expected to be supplied by the image, and the cost of the procedure. Thanks to the absence of electrical charge, neutrons and X-rays can penetrate deeper into matter than charged particles, being thus more suitable to examine thick metallic objects. Since neutrons and X-rays interact with matter in intrinsically different fashions, the materials will exhibit different attenuation coefficients for these radiations, furnishing hence different information about the object under analysis.

\section{Detector}

The gaseous Position Sensitive Detector - PSD, described in this work operates at the proportional region and is constituted by a gas chamber containing a multi-strip flat cathode. A wire placed between this cathode and the detector window acts as anode like in the conventional detectors. The electron avalanche produced near the anode induces a charge distribution in the cathode strips, which are connected to a delay line. The electronic signal produced by that charge distribution would propagate in both directions toward the extrem- 
ities of that line. A comparison of the time lapses required by both signals to arrive at the end of each line allows the determination of the travelled distances, defining thus the position where the ionizing event occurred [1].

The original device designed for X-ray detection had a carbon window and was filled with a standard mixture of $90 \%$ of argon and $10 \%$ of methane under a $2 \mathrm{~atm}$-pressure. To detect neutrons, it's nevertheless necessary to introduce into the detector a material with a high absorption cross section, capable of producing an ionizing radiation after its interaction with those particles.

Typical neutron-to-charged particle converters are ${ }^{6} \mathrm{Li}$, ${ }^{3} \mathrm{He}$ and ${ }^{10} \mathrm{~B} .{ }^{3} \mathrm{He}$ enriched helium and ${ }^{10} \mathrm{~B}$ enriched $B F_{3}$ are commonly used as filling gases for proportional detectors [2],[3]. In this work, to detect thermal neutrons, ${ }^{3} \mathrm{He}$ enriched helium has been utilized as filling gas.

\section{Detector Performance.}

The detector performance is characterized by quantitative features such as resolution, linearity and homogeneity [4]. The resolution is inferred from the full width at half maximum-FWHM of the Line Spread Function-LSF. The LSF is expressed by a curve of the fraction of registered radiation versus the distance along a line perpendicular to the radiation beam, whenever a narrow line-shaped source perpendicular to both is examined by an image acquiring system. Instead of a thin line, a more or less blurred image is obtained depending on the resolution for that particular system. In order to measure the detector resolution, a mask constituted by a foil of neutron absorber material provided with a slit collimator, is attached to the detector window. After the fitting of a Gaussian function to those experimental data, its FWHM is used to determine the Resolution ERS of the detector as follows:

$$
E R S=\sqrt{\left(F W H M^{2}-\Delta^{2}\right)}
$$

where $\Delta$ is the radiation beam width. When ${ }^{3} \mathrm{He}$ is used the proton and tritium produced by this reaction are isotropically emitted into opposite directions carrying 573 and 191 $\mathrm{keV}$ respectively. Each particle generates its own ionization track whose centroid is shifted from the point where the reaction occurred, due to the different particle ranges. Fig. 1 shows the behavior of the energy loss per unit of length $(d E / d x)$ along the particle track for proton and tritium produced by the nuclear reaction. This shift degrades the detector resolution as explained below. All ionization tracks centroids around a point where the nuclear reaction occurs are distributed over a spherical layer [5]. Disregarding the beam hardening, i.e., assuming a thin layer, the probability density for the centroids distributed along a parallel line to the anode wire is a rectangular function with a width limited by the external diameter of that spherical layer.

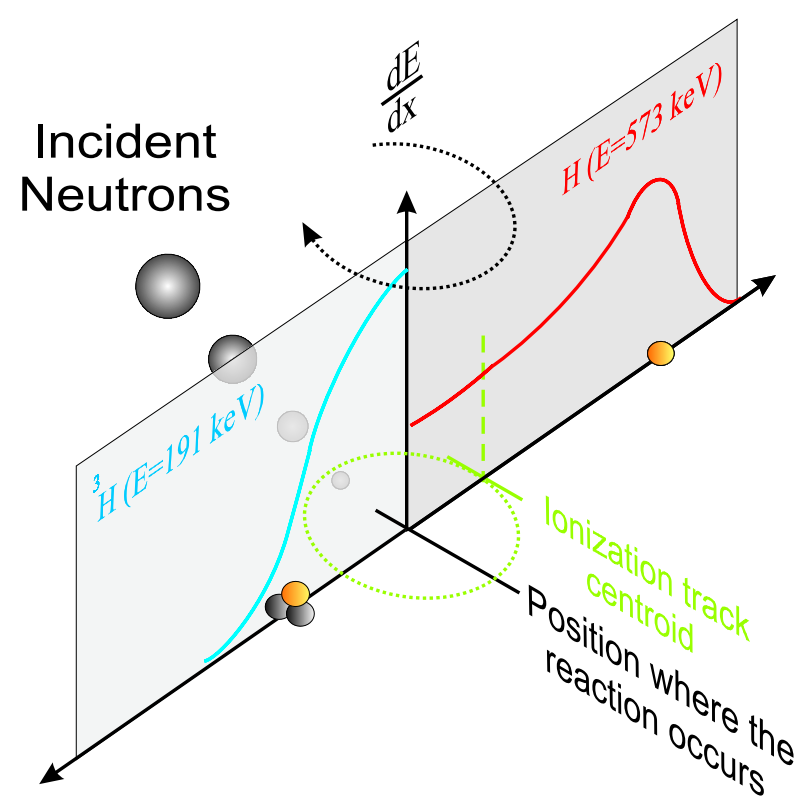

Figure 1. Specific energy loss along a proton track (right) and a tritium (left).

The position where a nuclear reaction occurs inside the detector is ultimately computed from the charge distribution induced on its cathode, and the centroid of this distribution is defined by the projection of the weigh center of all centroids on the cathode axis. Therefore when a narrow thermal neutron beam hits the detector window on a perpendicular direction the expected peak becomes flattened due to the above-mentioned phenomena, and hence the spatial resolution is degraded. Fig. 2 shows the measured resolution for the original PSD, as well as after its modification to detect thermal neutrons.

The linearity evaluates basically the quality of the delay line through the relationship between the actual position where an event occurs in the detector and the position registered by the system. This parameter can be measured by fitting to the detector window an opaque mask to the radiation, containing equally spaced slits. The radiation passing through these slits generates peaks defining the position where the events occur, as seen by the detector, while their actual positions are given by the position of the slits in the mask. The radioactive decay or the emission of radiation by an excited nuclide is a random process [2], and thus any measurement based upon the observation of the radiation emitted in that process is affected by a statistical fluctuation $\Delta \mathrm{N}$. A position sensitive detector can be roughly imagined as a set of single virtual detectors or cells. If each cell of the PSD is hit by neutrons during a time interval, such that $\mathrm{N}$ counts are produced, then this number will be affected by a probable uncertainty of $\sqrt{N}$. Uncertainties exceeding this figure should in principle be attributed to spoiling agents in the detection system. For a mono-energetic source, the homogeneity of a PSD is defined as the maximal deviation from the average counts obtained at all cells. If this number is large enough, the statistical fluctuation becomes insignificant and the non-homogeneity can be easily observed and assessed. For the two detectors analyzed in this work, the linearity and the homogeneity remained below $1 \%$ and $8 \%$ respectively. 


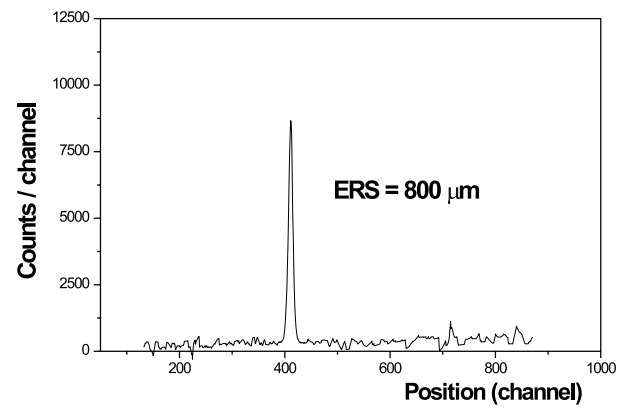

( a)

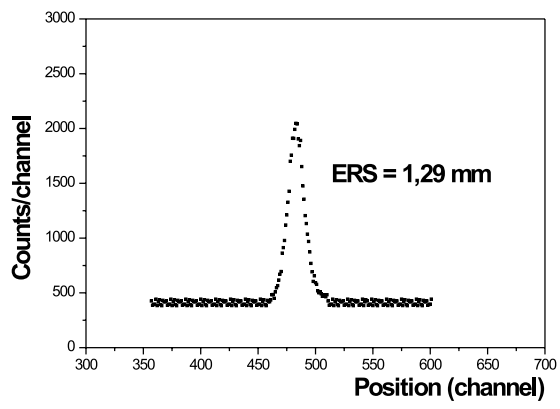

(b)

Figure 2. Resolution of the Position Sensitive Detector for X-Rays (a) and thermal neutrons (b).

\section{Tomographic systems}

The source employed at the X-ray tomographic system was a divergent beam produced by an ampoule working at a $25 \mathrm{kV}$ anode voltage being capable to yield a maximum current of $1 \mathrm{~mA}$. The maximum intensity of the typical bremsstrahlung spectrum, as determined by a berylliumwindow $\mathrm{NaI}(\mathrm{Tl})$ detector, occurs at an energy $\mathrm{E}$ (in $\mathrm{keV}$ ) which is a function of the applied anode voltage, as given by eq. (2), as follows:

$$
E=-6.55+1.21 V-0.014 V^{2}
$$

As already mentioned the Argonauta reactor of IEN was employed as source and furnished a flux of $4.5 \times 10^{5} \mathrm{n} \cdot \mathrm{cm}^{-2}$. $s^{-1}$ at the irradiation position where the tomography system is installed. The neutron beam has been made parallel, by using an arrangement incorporating graphite blocks and a cadmium honeycomb-type collimator.

\section{Results}

Images of different samples taken with $14 \mathrm{keV} \mathrm{X}$-rays and with $0.025 \mathrm{eV}$ neutrons using both tomographic systems are shown on Fig. 3. The samples consist of a $23 \mathrm{~mm}$-diameter cylinder provided with $3 \mathrm{~mm}$-diameter holes filled with some materials usually employed in engineering. For X-rays, a polyethylene cylinder has been used as matrix, while for neutrons it has been replaced by aluminium.

It can be observed that the highest contrasts are achieved with PVC, teflon and rubber, for the image taken with $\mathrm{X}$ rays, and with nylon, lucite, PVC and celeron for the image taken with thermal neutrons. The impact of the detector resolution on the quality of the images acquired by both tomographic systems can be observed in the Fig. 3. As previously stressed, the shift of the ionization track caused by the different ranges of the reaction products in the filling-gas spoils the detector resolution. When a comparison is done one can notice that both systems furnish contrasts consistent with the data plotted in Fig. 4. The complementary character of these radiations is clearly observed on Fig. 5.

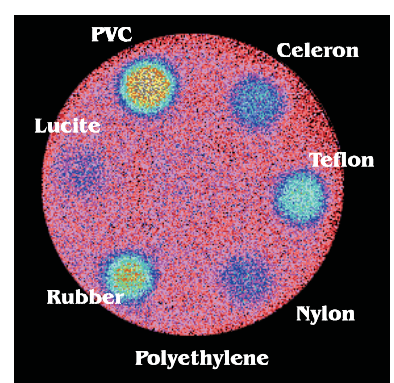

(a)

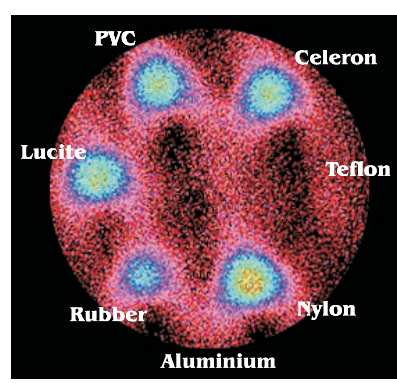

(b)
Figure 3. Tomographic images taken with a PSD. (a) X-rays (b)Thermal neutrons.

Indeed, samples which couldn't be properly analyzed by X-rays could be by thermal neutrons and vice-versa. A honeycomb type heat radiator used in the air-space industry, made of a $40 \mu$ m-thick aluminium sheet is well tomographed in spite of its small thickness. Such a thin aluminium sample wouldn't be ever caught by neutron tomography. On the other hand, samples like those shown on the right of the same figure, constituted by a teflon cylinder provided with orifices of several diameters, filled with gadolinium oxide and cadmium wires wouldn't be properly analyzed by X-ray due to the lack of contrast. Both cases therefore demonstrates not only the complementary character of both radiations but also the capability of the PSD to catch small objects and to resolve them for any of the above radiations.

One of the most useful tools to evaluate on a quantitative basis the performance of an image acquiring system is the Modulation Transfer Function-MTF. This function express how the ability of the system to distinguish single close features is impaired by decreasing the distance between them. Fig. 6 shows the MTF for the neutron tomographic system, experimentally determined by using a gadolinium collimator provided with slit apertures within the range 0.2 to $0.5 \mathrm{~mm}$, including the cutoff at $\frac{\delta x}{\delta 0}=0.1$. It's generally accepted that below that value the system is unable to reproduce an image with an acceptable quality [6]. For the system described in this work the cutoff value is reached at a $0.35 \mathrm{~mm}$-aperture. 


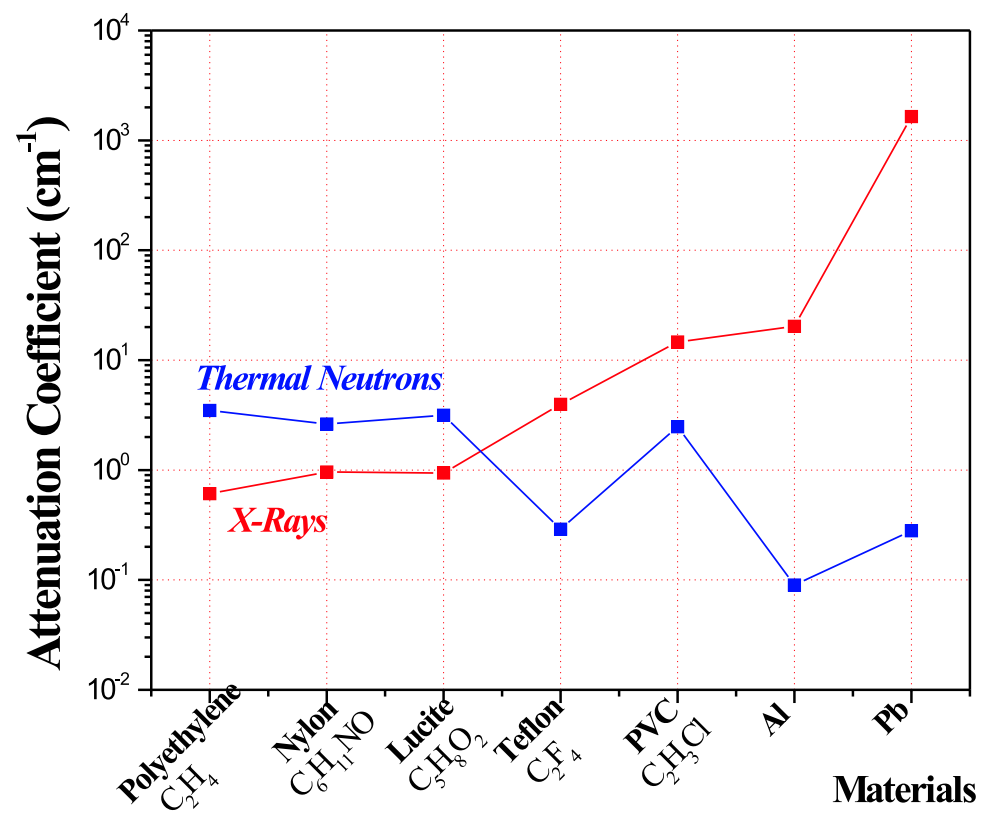

Figure 4. Comparison between the capabilities of X-rays and thermal neutrons to perform non-destructive assay in some materials, expressed by their attenuation coefficients.

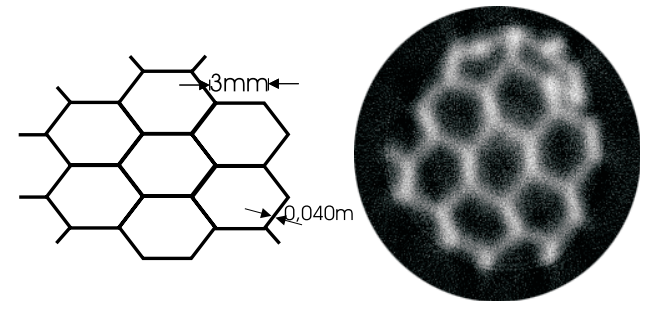

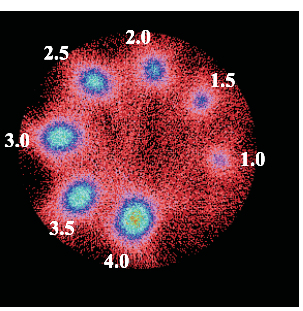

( a)

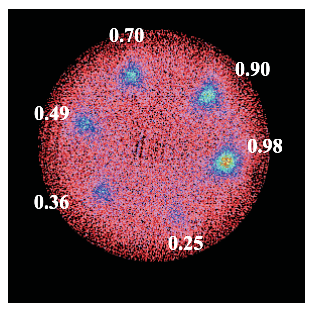

(b)

Figure 5. Left: Tomography of an Aluminum honeycomb-type radiator taken with X-rays. Right: Tomography of a teflon cylinder containing (a) Gadolinium oxide (b) Cadmium wires, both taken with thermal neutrons.

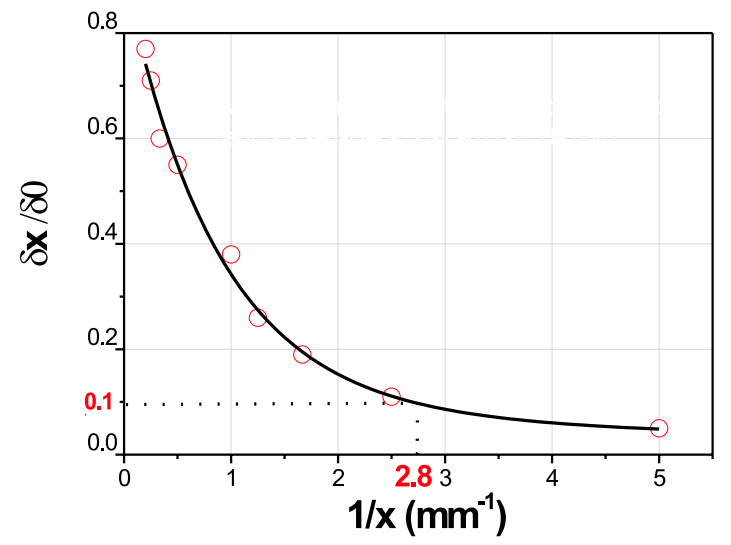

Figure 6. MTF curve for the Tomographic System equipped with a ${ }^{3} \mathrm{He}$-filled PSD. $\delta x$ and $\delta 0$ are the intensities of the transmitted and incident beam respectively. Continuous line is an exponential fitted to the experimental points.

\section{Conclusion}

X-ray and Thermal Neutron Non-Destructive assays are complementary techniques. Both can take advantage of the special features of a PSD, when incorporated to tomographic systems. A tight collimation is not needed to assure an acceptable image quality as in conventional systems. Therefore, an equivalent resolution can be achieved with beams of lower intensity and/or less acquisition times.

A sample translation is no longer required and thus the acquisition time is substantially reduced. The obtained results encourages further efforts towards the design of a specific neutron-tailored detector, aiming the improvement of both efficiency and resolution. Further details can be found in [7].

\section{Acknowledgements}

The authors would like to thank the CNPq for the financial support. 


\section{References}

[1] A. Gabriel, Rev. Sci. Instr. 10, 1303 (1980).

[2] G. F. Knoll, 1989, Radiation Detection and Measurement, ed. 2, USA, John Wiley and Sons.

[3] D. Reilly, N. Ensslin, H. Jr. Smith, 1991, Passive Nondestructive Assay of Nuclear Materials, 1st ed., USA, Sarah Kreiner.

[4] A. F. Barbosa. Report CBPF-NF-068/94, (1994).
[5] J. Alberi, J. Fischer, V. Radeka, L.C. Rogers and B. Schoenborn. Nucl. Instr. and Meth. 127, 507 (1975).

[6] ASTM E 1441-95 and 1570-95a, 1996, ” Non-Destructive Testing, Radiation Methods, Computed Tomography, Guide for Imaging and Practice for Examination", ISSO/TC 135/SC 5, N118

[7] M. I. Silvani, "Computed Tomography with thermal neutrons and a Position Sensitive Detector," DSc. Thesis, COPPE/UFRJ-Brazil 2001 (In Portuguese). 